\title{
Highly Sensitive Sensors Based on Photonic Crystal Fiber Modal Interferometers
}

\author{
Joel Villatoro, ${ }^{1}$ Vittoria Finazzi, ${ }^{1}$ Gonçal Badenes, ${ }^{1}$ and Valerio Pruneri ${ }^{1,2}$ \\ ${ }^{1}$ Institut de Ciencies Fotoniques (ICFO), Mediterranean Technology Park, Castelldefels, 08860 Barcelona, Spain \\ ${ }^{2}$ Institució Catalana de Recerca i Estudis Avançats (ICREA), 08010 Barcelona, Spain
}

Correspondence should be addressed to Joel Villatoro, joel.villatoro@icfo.es

Received 23 March 2009; Accepted 19 May 2009

Recommended by Christos Riziotis

We review the research on photonic crystal fiber modal interferometers with emphasis placed on the characteristics that make them attractive for different sensing applications. The fabrication of such interferometers is carried out with different post-processing techniques such as grating inscription, tapering or cleaving, and splicing. In general photonic crystal fiber interferometers exhibit low thermal sensitivity while their applications range from sensing strain or temperature to refractive index and volatile organic compounds.

Copyright (c) 2009 Joel Villatoro et al. This is an open access article distributed under the Creative Commons Attribution License, which permits unrestricted use, distribution, and reproduction in any medium, provided the original work is properly cited.

\section{Introduction}

Optical interferometers have played an important role in both fundamental and applied research during the past two centuries. The famous Young's double-slit experiment, for example, provided experimental support to the wave theory of light which had important consequences in physics, optics, and science in general. On the other hand, Abraham Michelson demonstrated the high resolution of an interferometer when it is used for metrology applications.

Fiber optics technology offers many degrees of freedom and some advantages such as stability, compactness, and no moving parts, for the construction of different types of interferometers [1-3]. Different types of fibers exist and can be used to build interferometers. Two different approaches are commonly followed. One consists of splitting and recombining two monochromatic optical beams that propagate in different fibers. These two-arm interferometers typically require several meters of optical fibers and one or two couplers [1-3]. The other approach consists of exploiting the relative phase displacement between two modes, typically the first two modes like the $\mathrm{LP}_{01}$ and $\mathrm{LP}_{11}$, or the $\mathrm{HE}_{11}$ and $\mathrm{HE}_{21}$. Interferometers based on the latter approach are known as modal interferometers. These have inherent advantages when compared to their two-arm counterparts. Since the modes propagate in the same path, the susceptibility to environmental fluctuations is reduced. In addition, one requires controlling only two or more modes. The compactness and simplicity of modal interferometers have gained considerable attention in many research groups. Here we review the different alternatives reported so far to construct all-fiber modal interferometers with photonic crystal fibers (PCFs). These fibers are characterized by a complex pattern of microscopic air-holes in the transverse plane that runs all over the fiber $[4,5]$. The holey structure gives PCFs unique guiding mechanisms and modal properties that are not possible with conventional optical fibers. For this reason we focus only on PCF modal interferometers.

The unique properties of PCFs have intrigued the sensor community. Many research groups around the world have investigated different schemes which exploit the properties or structure of PCFs with a view to developing new optical sensors. Like a conventional optical fiber the propagation properties of a PCF can be modulated by external parameters such as strain, temperature, and pressure. However, they can also be modulated with liquids or gases infiltrated into the voids of the PCF, see for example [6]. It is, therefore, desirable to investigate new schemes which exploit the properties of PCFs with a view to developing new optical sensors. The construction of PCF-based interferometers in particular is interesting owing to their proven high sensitivity 

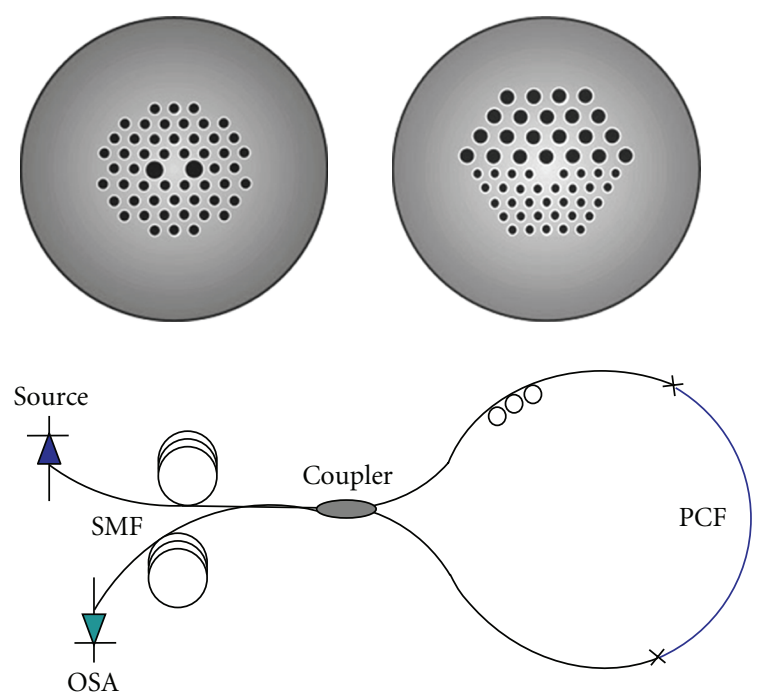

Figure 1: The top figures show the cross-sections of polarization maintaining PCF (top-left) or high birefringence PCF (top-right), taken from [6]. The bottom drawing is a diagram of the Sagnac interferometer. OSA stands for optical spectrum analyzer, SMF for single mode fiber, and PCF for photonic crystal fiber.

and their broad range of applications. To the authors' best knowledge, the first attempt to construct an interferometer with a PCF was reported in 2001 by MacPherson et al. [7]. They used a dual-core photonic crystal fiber in which the two cores played the role of arms of a Mach-Zehnder interferometer. Since then other authors have reported a variety of interferometers built with different types of PCFs in which the relative phase displacement between two modes is exploited.

Two modes can be excited in a short piece of PCF or standard optical fiber under critical launching and polarization conditions which is impractical for optical sensing [813]. To overcome these drawbacks, the microstructure of the PCF can be designed to support only two modes [14], or to use long period gratings, tapering, or splicing techniques. The interesting features of PCF modal interferometers built with the later techniques are compactness, low temperature sensitivity, broad operation wavelength range, and high stability over time. All these properties are important for unambiguous measurement of the interferometer phase, and hence the parameter being sensed. The different approaches to build compact modal interferometers with PCFs will be reviewed in the following paragraphs placing emphasis on the characteristics that make them attractive for different sensing applications.

\section{PCFs in Fiber Loop Mirrors}

One configuration that has been widely explored consists of a structure where a relatively short length of polarization maintaining $(\mathrm{PM})$ or high birefringence (Hi-Bi) PCF is inserted in a fiber loop mirror, see Figure 1. This configuration is also known as Sagnac interferometer. Basically, in this

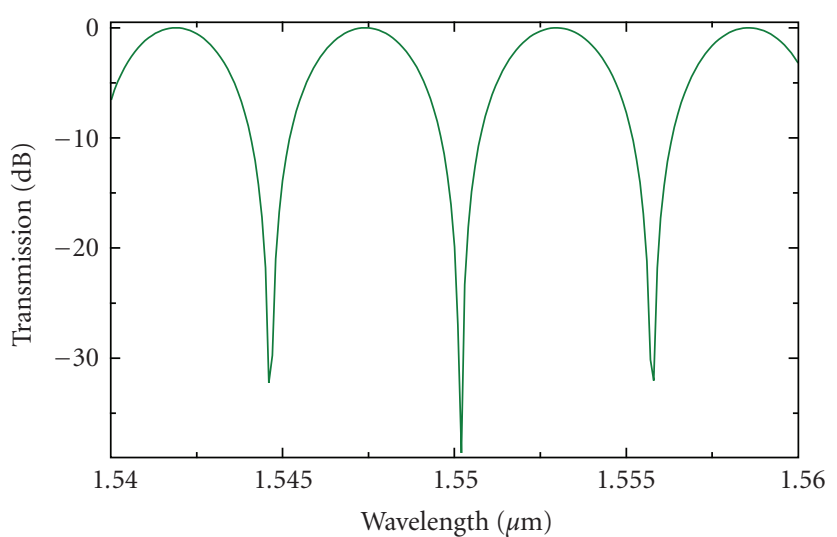

(a)

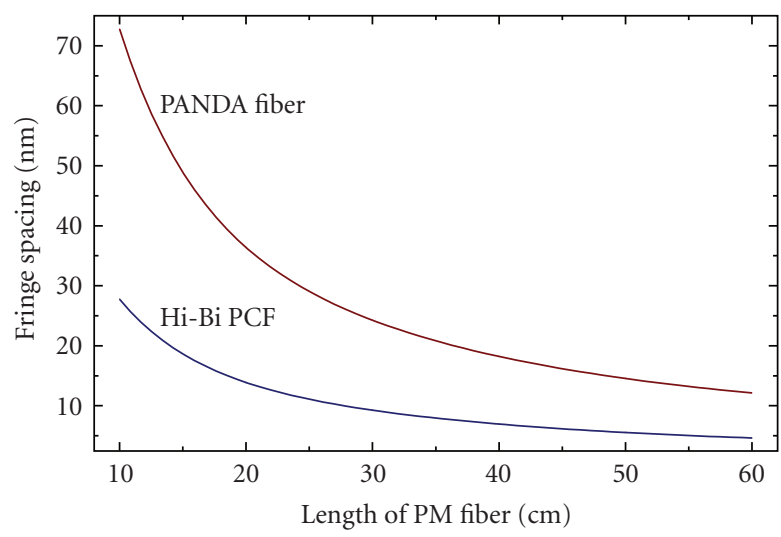

(b)

Figure 2: (a) Theoretical transmission spectrum of a Sagnac interferometer built with $500 \mathrm{~mm}$ of Hi-Bi PCF described in [15]. (b) Fringe spacing as a function of the length of fiber. The period of a Sagnac interferometer built with PANDA fiber is also shown for comparison.

interferometer the input wave is split into two by the $3-\mathrm{dB}$ coupler. The two modes counterpropagate through the same waveguide and then they are subsequently recombined by the same coupler. The resulting transmission spectrum is determined by the relative phase difference introduced to the two orthogonal guided modes. The phase difference between the modes depends on the wavelength, the group birefringence, and the length of the HiBi or PM fiber. The interference of the counterpropagating modes will be constructive or destructive, depending principally on the birefringence of the waveguide. The transmission spectrum of the Sagnac interferometer is approximately a periodic function of the wavelength. The average wavelength spacing $(\Delta \lambda)$ between consecutive transmission peaks is $\Delta \lambda \approx \lambda^{2} /\left(\Delta n_{g} L\right)$, being $\lambda$ the wavelength, $\Delta n_{g}$ the group birefringence of the HiBi fiber, and $L$ the length of $\mathrm{Hi}-\mathrm{Bi}$ or PM PCF. An additional advantage of the Sagnac interferometer is that it typically exhibits high extinction ratio which is useful to track the position of the interference peaks.

Figure 2 shows the theoretical transmission spectrum over $20 \mathrm{~nm}$ of a Sagnac interferometer built with $50 \mathrm{~cm}$ 
of commercially available Hi-Bi PCF which has $\Delta n_{g}=$ $8.65 \times 10^{-4}$ for $\lambda=1550 \mathrm{~nm}$, see [15]. The calculated fringe spacing as a function of the length of PCF is also shown. For comparison the corresponding $\Delta \lambda$ for a Sagnac interferometer built with PM fiber, commercially known as PANDA fiber $\left(\Delta n_{g}=3.3 \times 10^{-4}\right.$ for $\left.\lambda=1550 \mathrm{~nm}\right)$, is also given in the figure. Since the birefringence of Hi-Bi PCFs is in general larger than that of conventional Hi-Bi fibers then Sagnac interferometers will exhibit much shorter periods than their counterparts built with standard optical fibers. This property helps to construct more compact Sagnac interferometers with PCFs.

Note that the two interfering modes in the Sagnac interferometers are exposed to the same environment. This makes the interferometer insensitive to ambient temperature. Unlike conventional polarization maintaining fibers (bowtie, elliptical core, or PANDA), which contain at least two different glasses each with a different thermal expansion coefficient, thereby causing the polarization of the propagation wave to vary with temperature, the PCF birefringence is highly insensitive to temperature because it is made of a single material. Therefore temperature-insensitive Sagnac interferometers built with PCFs are expected. The experiments carried out independently by different researchers confirmed low temperature sensitivity $\left(\sim 0.3 \mathrm{pm} /{ }^{\circ} \mathrm{C}\right)$ in this type of interferometer $[15,16]$. The studies carried out on the thermal sensitivity of Sagnac interferometer revealed that Hi-Bi and PM PCFs had, respectively, 30 and 45 times smaller temperature dependence than that of their conventional fiber counterparts $[15,16]$.

The development of temperature-insensitive sensors based on PCF Sagnac interferometers was natural. So far different sensors for physical parameters such as strain, pressure or curvature had been demonstrated [15-20]. The sensitivities of these sensors depend critically on the type of PCF. For example, strain sensors exhibit sensitivities ranging from 0.23 to $1.2 \mathrm{pm} / \mu \varepsilon[16,17]$ while their thermal sensitivity is really low, of the order of $0.3 \mathrm{pm} /{ }^{\circ} \mathrm{C}$. However, when compared to other fiber-based strain sensors PCF Sagnac interferometers probably are not really competitive. They require several centimeters of PCF, from 8.6 to $56 \mathrm{~cm}$, which is nearly an order of magnitude longer than that of the popular fiber Bragg grating strain sensors. Another disadvantage is the high loss caused when splicing $\mathrm{Hi}$ $\mathrm{Bi}$ PCFs to standard optical fiber and the intrinsic losses of the PCF. The overall losses can be of the order of $10 \mathrm{~dB}$.

Recently, a novel Hi-Bi photonic crystal fiber consisting of half region composed by large diameter holes and the other half containing small diameter holes (see Figure 1) was proposed for bend sensing in a Sagnac interferometer [20]. It was found that the sensor exhibited maximum sensitivity when the large holes were compressed. Insensitivity to temperature and longitudinal strain were also observed. Also a photonic band gap PCF with elliptical core was demonstrated in a Sagnac interferometer for strain and temperature sensing [21]. These recent works reflect the variety of possibilities that PCFs offer to construct Sagnac interferometers.

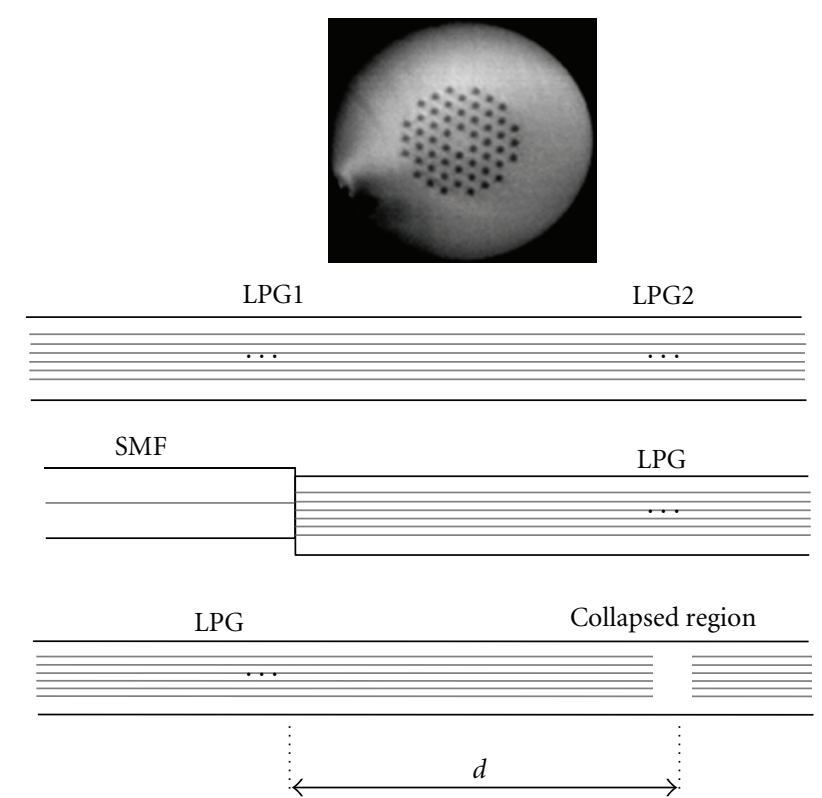

Figure 3: Micrograph of the cross-section of the typical PCF employed to build interferometers with LPGs. Diagrams of interferometers based on two LPGs in series, a misaligned point end together with an LPG, and an LPG together with a collapsed region in the PCF. The separation between the splitting gratings and recombining element is denoted by $d$.

\section{Interferometers Built with Long Period Gratings}

A long period grating (LPG) is a periodic modulation of the core refractive index whose period is much larger than the wavelength [22]. LPG are used to induce the mode coupling between a codirectional propagating core mode $\left(\mathrm{LP}_{01}\right)$ and cladding modes $\left(\mathrm{LP}_{0 m}\right)$, where $m=2,3,4, \ldots$ at discrete wavelengths. The transmission spectrum of an LPG exhibits dips at the wavelengths corresponding to resonances with various cladding modes. The resonance wavelength $\lambda_{r}$ depends on the period of the LPG (usually denoted by $\Lambda$ ) and the difference between the effective refractive indices of the core and cladding modes. Since these modes propagate at different phase velocities, therefore, in-fiber interferometers can be implemented with a pair of LPGs-they were first studied using conventional optical fibers, see for example [23-30]. A popular configuration is to use two identical LPGs in cascade, see Figure 3. The first LPG couples part of the core mode power into a forward-propagating cladding mode and the second LPG recombines the two modes. The two LPGs function as beam-splitter/combiner and the core and the cladding modes travel through two independent paths along the same fiber. The resulting interference pattern of an interferometer formed with two LPGs in series exhibits sharp interference fringes. To a good approximation the fringe spacing $(\Delta \lambda)$ can be given as $\Delta \lambda=\lambda_{r}^{2} /\left(\Delta n_{\text {eff }} d\right)$, where $\Delta n_{\text {eff }}$ is the effective index difference between core and cladding modes and $d$ the separation between the gratings [24]. 
The idea of constructing interferometers with LPGs has been extrapolated to PCFs, but unlike conventional Gedoped single-mode fibers, in which UV-induced LPGs can be fabricated, PCFs have no photosensitivity because they are composed of pure silica. Therefore new ways to fabricate interferometers with LPGs in PCFs had been explored. The essential idea behind an LPG-based interferometer is that half of the fundamental $\mathrm{LP}_{01}$ core mode should be coupled to a cladding mode at the first LPG and half of the cladding mode should be coupled back to the core mode at the second LPG. Thus, for an all-PCF interferometer, one has to form two 3-dB LPGs with identical transmission spectra. It is difficult to form two identical 3-dB LPGs not only in PCF but also in standard optical fiber.

One of the approaches to achieve LPGs in photonic crystal fiber consists of pressing a section of the PCF with a periodic grooved plate $[31,32]$. The length and period of the grooved plate as well as its width determines the transverse pressure applied on the PCF. Periodic pressure on the fiber surface induces periodic index changes in the fiber. The efficiency of the mode coupling between the core mode and a cladding mode varies with pressure. LPGs with proper lengths and periods have to be selected such that mode coupling occurs at the predetermined wavelength $[31,32]$. The advantage of this type technique is that it is easy to form two identical LPGs, and therefore, it is easy to control the properties of the interferometer. However, the control is manual which severely limits the reproducibility of the devices.

As an alternative to the above approach a combination of a misaligned splicing point (MSP) and an LPG was proposed to form all-PCF interferometers [32]. The MSP can be formed, for example, by using a commercial fusion splicer in manual operation. The MSP acts as a light splitter which couples a part of the core-mode power into the cladding modes so that it may replace the first LPG in an LPG pair to form an interferometer. In principle the formation of the MSP is easier, moreover, it is permanent. Another alternative that was proposed consists of combining a single LPG and a short region of the PCF in which the airholes are collapsed [33]. In this case the LPG was imprinted with the electric arc discharge of a fusion splicer by using the point-bypoint technique. The air holes of the PCF collapsed over a microscopic region behaves like a recombining element. Since the LPG and the collapsed section are permanent very sensitive interferometers can be effectively implemented. By adjusting the separation between the collapsed region and the LPG the spectral properties of the interferometer, specifically the interference fringe spacing, can be easily controlled. This type of interferometer was investigated for strain sensing applications. A sensitivity of $1.8 \mathrm{pm} / \mu \varepsilon$ was found which is slightly higher that the typical sensitivity of an $\operatorname{FBG}(1.2 \mathrm{pm} / \mu \varepsilon)$ [33].

More recently the fabrication of LPG-based PCF interferometers was demonstrated in which the gratings are inscribed by using a high-frequency $\mathrm{CO}_{2}$ laser [34]. By controlling the coupling coefficients of the two LPGs to be $3 \mathrm{~dB}$, sharp interference fringes due to coherent mixing of the core and the cladding mode were obtained around the resonant wavelength of the LPGs. The temperature and strain sensitivities for the interferometers were measured. The former was found to be equal to $42.4 \mathrm{pm} /{ }^{\circ} \mathrm{C}$ per meter which suggests that with a separation between the two LPGs of $25 \mathrm{~mm}$ the interferometer can exhibit sensitivity of only $1.06 \mathrm{pm} /{ }^{\circ} \mathrm{C}$. The strain sensitivity was found to be $\sim 2.6 \mathrm{pm} / \mu \varepsilon$ which is higher than that of a Sagnac interferometer or that of FBGs.

It should be pointed out that the type of PCF preferred for LPG-based interferometers is the so called endlessly single mode PCF whose cross-section is shown in Figure 3. In this PCFs $\Delta n_{\text {eff }} \approx 3 \times 10^{-3}$ at $1550 \mathrm{~nm}$ which suggests that $\Delta \lambda$ can be in the range between 27 to $5.5 \mathrm{~nm}$ when the separation $d$ between the gratings varies from 3 to $15 \mathrm{~cm}$. This means that compact interferometers can be fabricated with LPGs with the advantage that no coupler is required. Some drawbacks of LPG-based PCF interferometers are the relatively high insertion loss. The later originates from the loss caused by the splicing of the PCF with the SMF, the deformation of the PCF microstructure to inscribe the gratings, the misaligning or collapsed point, and so forth. All these factors also give rise to interferometers with slightly distorted interference fringes. This may make difficult the positional tracking of the interference peaks or dips which is crucial in sensing applications.

\section{Interferometers Built Tapered PCFs}

PCF combined with tapering technology can result in compact interferometers with interesting features. Tapering basically consists of stretching the fiber while it is heated over a localized zone. When tapering a PCF the diameter of the fiber and the relative size of the airholes can be scaled down, depending on the PCF design these variations have multiple applications, see, for example, [35-39]. During the tapering the voids of the PCF can be intentionally collapsed around the heated zone which allows interferometers with interesting features. This idea was demonstrated some years ago by one of the present authors and collaborators [4045]. By collapsing the air holes, a short zone of the PCF is transformed into a solid unclad multimode optical fiber, see Figure 4. As a consequence, the fundamental mode of the PCF is coupled to the modes of the solid fiber. The beating between the modes makes the transmission of the taper versus wavelength to exhibit an oscillatory pattern. By controlling the taper diameter and its length one can tailor the performance of the interferometer in a similar manner than those based on tapered conventional fibers [46, 47]. Tapering dual core fiber or photonic bandgap PCF also allows the development of interferometers $[48,49]$ but in these cases the voids of the PCF are not collapsed.

The interference patterns observed in tapered PCFs with collapsed airholes is a consequence of a phenomenon of mode coupling and beating. The single-mode holey fiber is gradually transformed into a solid unclad multimode fiber of diameter $\rho_{w}$ and length $L_{0}$ (or viceversa), see Figure 4 . In the contracting zone of the taper the fundamental $\mathrm{HE}_{11}$ mode of the PCF couples to the $\mathrm{HE}_{1 m}$ modes supported 

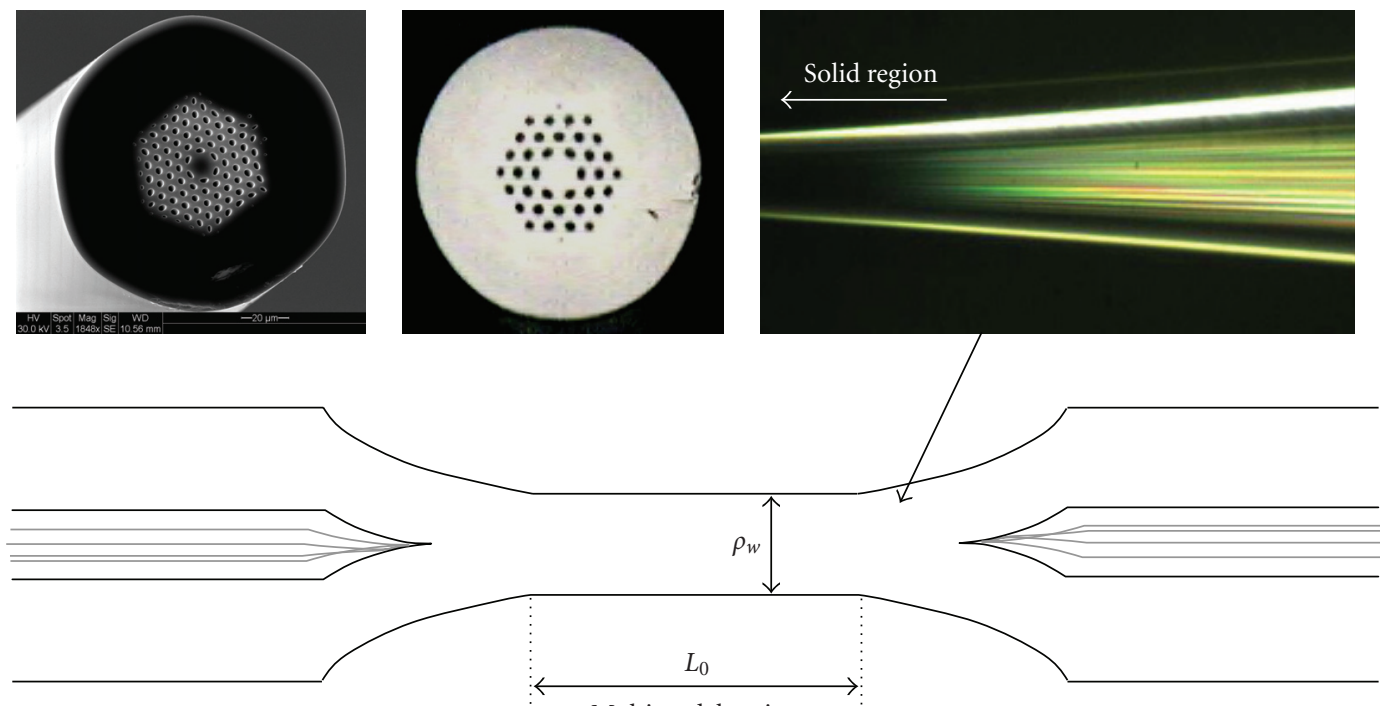

Multimodal region

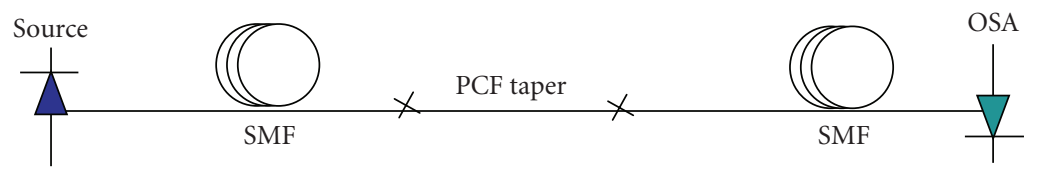

Figure 4: The top micrographs are the cross-section of some PCFs employed to fabricate tapers and the lateral view of the expanding zone of a taper. The middle drawing is a diagram of interferometers built with tapered PCFs. $L_{0}$ and $\rho_{w}$ are, respectively, the length and width of the taper waist. The bottom drawing represents the transmission measuring setup.

by the multimode fiber. The $\mathrm{HE}_{1 m}$ modes have propagation constants $\beta_{1 m}$. In the region of the tapered PCF of constant diameter the $\mathrm{HE}_{1 m}$ modes beat or interfere but no coupling occurs. After propagating such a beating length the modes accumulate phase difference. The expanding zone of the taper recombines the multiple modes of the solid fiber to core modes of the PCF. Therefore, a tapered PCF with collapsed air-holes can be considered as an interferometer. The contracting and expanding zones are equivalent to couplers or beam splitters and the modes of the beating region can be considered as arms of the interferometer. The transmission spectra of these modal interferometers also exhibit a series of maxima and minima, analogous to those of the Sagnac interferometers or the LPGs-based ones. However, for thick tapers $\left(\rho_{w}>15 \mu \mathrm{m}\right)$ the interference pattern does not vary sinusoidally with wavelength [40-44]. If the PCF is tapered down to diameters between 3 to 5 microns, then only two higher-order modes ( $\mathrm{HE}_{05}$ and $\left.\mathrm{HE}_{06}\right)$ survive [45]. The fringe spacing $(\Delta \lambda)$ in this case is $\sim \Delta \lambda=$ $\lambda^{2} /\left(\Delta n_{\text {eff }} L_{0}\right)$, being $\lambda$ the wavelength of the source, $\Delta n_{\text {eff }}$ the effective index difference between the modes participating in the interference and $L_{0}$ can be taken as the length of the taper waist. For two higher-order modes $\Delta n_{\text {eff }}$ is large; therefore, the period of the interference pattern of a PCF microtaper can be very short, even for compact devices.

In PCF microtapers $\Delta n_{\text {eff }} \approx 0.5$ which is about two orders or magnitude larger than $\Delta n_{\text {eff }}$ or $\Delta n_{g}$ of LPG-based or Sagnac PCF interferometers, as mentioned above.

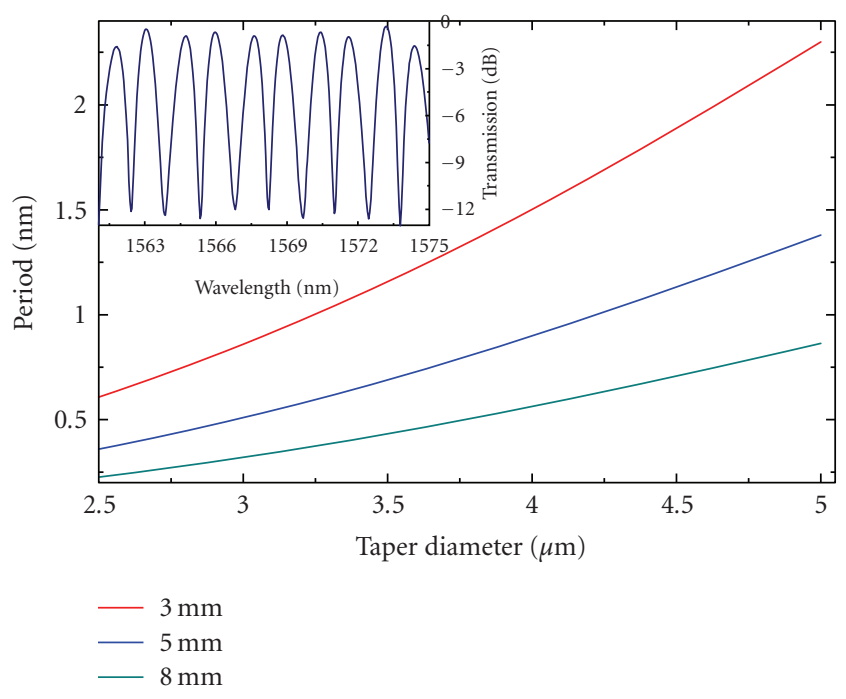

FIGURE 5: Predicted period of the interferometers at $1550 \mathrm{~nm}$ as a function of the diameter of the micro tapers for different lengths of their waist. The inset shows the experimental transmission spectrum of a $5 \mu \mathrm{m}$-thick taper in which the average fringe separation is $1.4 \mathrm{~nm}$ and the width of the fringes is around $0.9 \mathrm{~nm}$.

Figure 5 shows the estimated period as a function of the taper diameter for some values of $L_{0}$. It can be noted that interferometers with nanometer and even sub-nanometer 


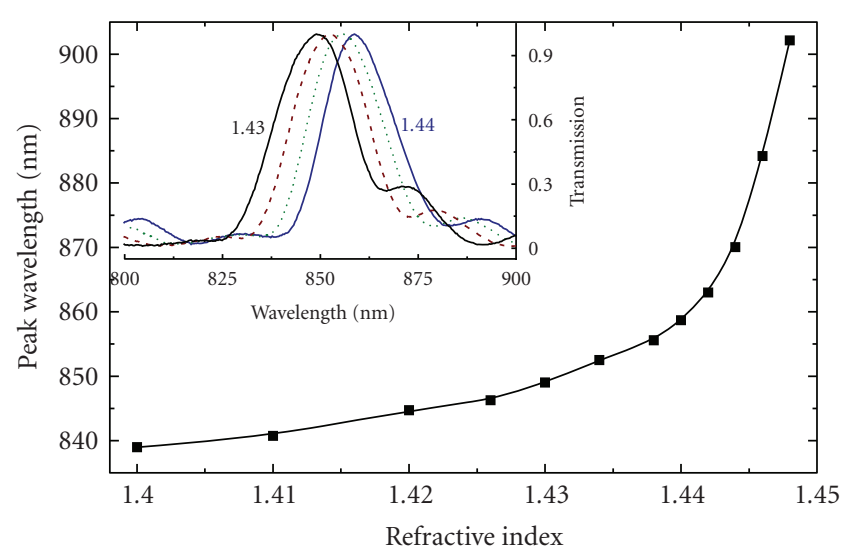

Figure 6: Position of the peak as a function of the external index observed in a $30 \mu \mathrm{m}$-thick taper. (Inset) Transmission spectra around $850 \mathrm{~nm}$ of the taper immersed into Cargille oils with indexes of $1.430,1.434,1.438$, and 1.440 .

fringe spacing can be fabricated. The figure, for example, shows the transmission spectrum observed in a $5 \mu \mathrm{m}$-thick device in which the fringe spacing is only $1.4 \mathrm{~nm}$, that is, a $\sim \lambda / 1100$. At shorter wavelengths, the fringes are even closer. These features are important for sensing applications since narrow fringes or peaks help to improve the accuracy and resolution of any sensor.

The modes in PCF tapers are sensitive to the external medium. The interference pattern can be shifted if the external refractive index changes which suggest that the tapers can be used for refractometric applications [40]. In the inset of Figure 6 we show the observed transmission spectra of a $30 \mu \mathrm{m}$-thick taper for different refractive indexes of the external medium. The figure also shows the position of the highest peak as a function of the index. It can be noted that the device exhibits larger shifts as the index of the external medium approaches that of the PCF. In the 1.435-1.448 range the shift is $\sim 50 \mathrm{~nm}$ which suggests that a resolution of the order of $3 \times 10^{-5}$ can be achieved (assuming that a shift of $100 \mathrm{pm}$ can be resolved). At longer wavelength the resolution may be higher [40] but the advantage at shorter wavelengths (around $850 \mathrm{~nm}$ ) is that cost-effective light sources and spectrometers are commercially available. The results shown in Figure 6 demonstrate that PCF taperbased refractive index sensors can compete with others based on other technologies.

If a tapered PCF is subjected to strain the interference pattern also shifts, thus making possible the development of strain sensors [41]. The sensitivity of these strain sensors is considerably high $(\sim 5.3 \mathrm{pm} / \mu \varepsilon)$ while the dynamic range is really broad. Strain up $8000 \mu \varepsilon$ was demonstrated in [41]. The temperature sensitivity was found to be of the order of $12 \mathrm{pm} /{ }^{\circ} \mathrm{C}$. A remarkable feature of PCF tapers is that they can withstand ultra-high temperature, up to $1000^{\circ} \mathrm{C}$, with minimal degradation thus making possible the sensing of high temperature [42].

In addition to physical parameters taper-based PCF interferometers can also be exploited for gas and chemical

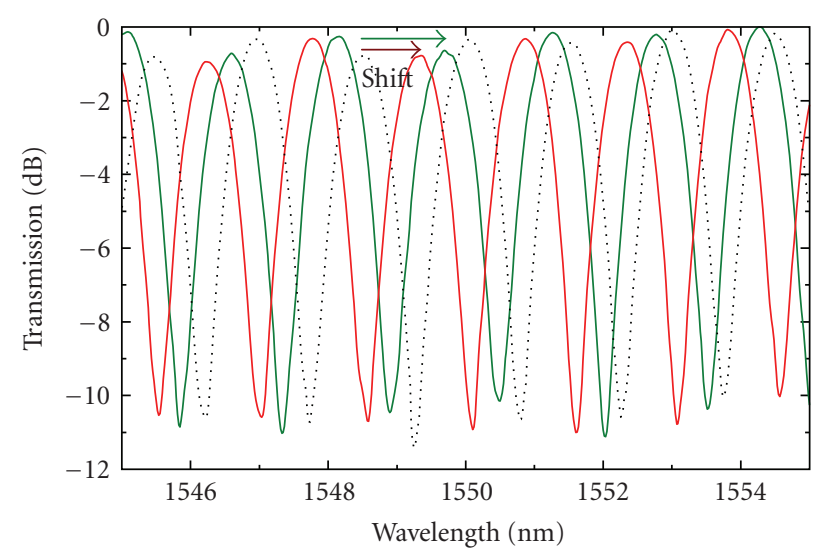

Figure 7: Normalized transmission spectra of an interferometer with diameter of $5 \mu \mathrm{m}$ and waist of $5 \mathrm{~mm}$ in length when the external media were air (dotted line), vapor molecules of isopropyl alcohol (red line), and vapor molecules of acetone (green line). The arrows indicate the direction of the shift.

sensing since the evanescent waves of the modes reach the external environment [43]. The solid region of the taper can be coated with gas-permeable layers thus making possible the detection of different gases. The detection of low concentration of hydrogen at room temperature was demonstrated in which a thin layer of Pd was deposited on the taper waist. More recently it was demonstrated that if a PCF consisting of 3 rings of air holes is tapered down to 3 to 5 microns, the fiber becomes sensitive to some chemical compounds without the need of any permeable material [45]. Figure 7, for example, shows the response of a $5 \mu \mathrm{m}$-thick taper in air and when it was exposed to vapors of isopropyl alcohol and acetone. It can be observed that the interference patterns shift to longer wavelengths. A red shift was also observed when the taper was exposed to vapors of ethyl and methyl alcohol, methylene chloride, and chloroform [45]. In all cases the interference pattern was returned to its original position when the VOCs were removed.

\section{Interferometers Built via Micro-Holes Collapsing}

More recently, new interferometers based on microhole collapse have been demonstrated [50-55]. This technique is really simple since it only involves cleaving and splicing, processes that can be carried out in any fiber-optics laboratory. The key element in these interferometers is a microscopic region in which the voids of the PCF are fully collapsed. Basically, the collapsed region is what allows the excitation of two modes in the PCF. The appeal of the interferometers fabricated with this approach is that the devices can be used for a variety of applications ranging from sensing strain (and all the parameters that can be translated to strain) or temperature to refractive index (biosensing) and volatile organic compounds (VOCs). In addition, the devices are compact, robust, and highly stable over time. For their interrogation 

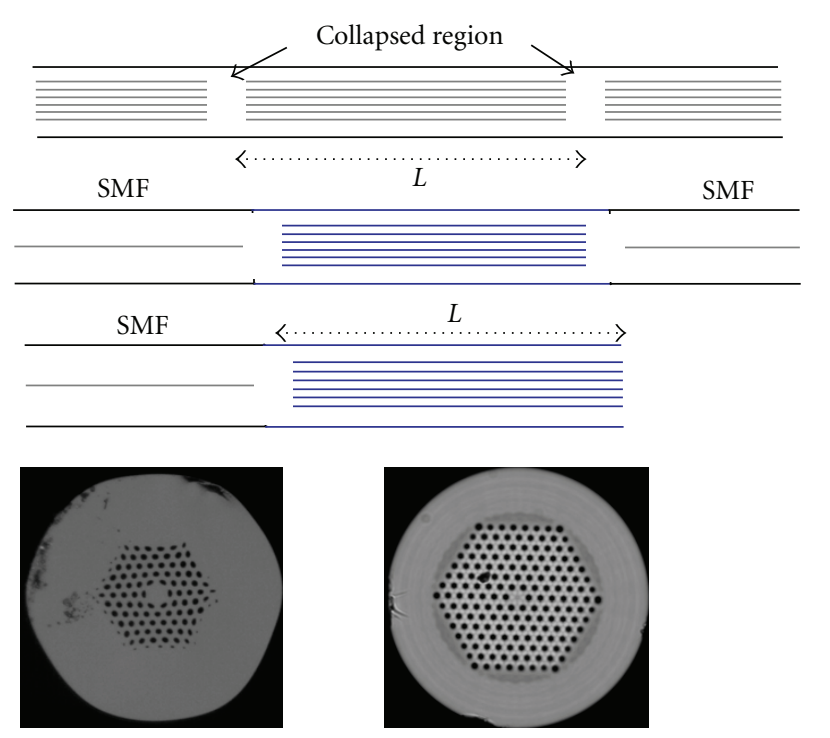

Figure 8: Diagrams of PCF interferometers built via microhole collapsing. The bottom images are the cross section of some PCFs employed in this type of interferometers. $L$ is the length pf PCF or length between the collapsed regions.

a light emitting diode (LED) and a conventional fiber Bragg grating (FBG) interrogator or spectrum analyzer or a tunable laser and a single photodetector can be employed.

It is known that when splicing together two PCFs, or a PCF and a conventional optical fiber, with the standard electric-arc method, the air-holes of the PCF collapse completely in the vicinity of the splice $[56,57]$. The length of the collapsed region is typically less than 300 or $400 \mu \mathrm{m}$. This is not a serious drawback of fusion splices since it may introduce minimal losses. The collapsing of the voids in a short region makes the fundamental PCF mode to spread out since it reaches a piece of coreless fiber, see Figure 9. The broadening of the fundamental mode can be estimated by using a Gaussian beam approximation [56]. According to it the fiber's mode field diameter (MFD) at any $z$ (the direction of propagation) depends on the wavelength $(\lambda)$ of the guided light, the refractive index $n_{1}$ of the medium (pure silica in our case), and the light spot size $\left(\omega_{0}\right)$ as

$$
\operatorname{MFD}=2 \omega_{0} \sqrt{1+\left(\frac{z \lambda}{n_{1} \pi \omega_{0}^{2}}\right)^{2}} .
$$

If we assume that the solid region starts at $z=0$, see Figure 9, then at the end of the solid region, let us say at $z_{1}=300 \mu \mathrm{m}$, according to (1), the MFD practically quadruplicates when $\lambda=1.55 \mu \mathrm{m}$ and triplicates when $\lambda=0.85 \mu \mathrm{m}$. This means that the enlarged mode does not reach the surface of the collapsed region and it is therefore insensitive to the external medium. At $z \sim 300 \mu \mathrm{m}$ the enlarged mode reaches a piece of PCF of length $L$ in which two modes can be excited. In Figure 9, for example, we show the transverse component of the electric field of two core modes. The calculations were performed using finiteelement method (COMSOL Multiphysics) applied to the

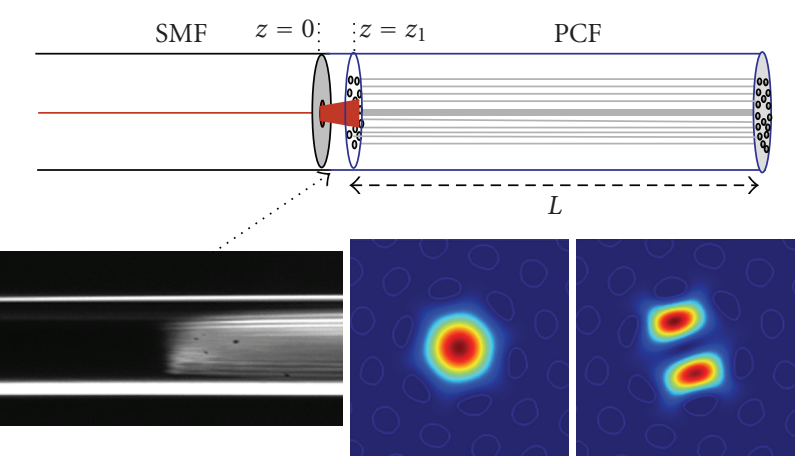

FIGURE 9: Diagram of SMF-PCF splice illustrating the broadening of the fundamental mode. The bottom images are a micrograph of the PCF in the vicinity of the splice and the transverse components of the electric field of the $\mathrm{LP}_{01}$ - and $\mathrm{LP}_{11}$-like modes of a real PCF.

index profile based on the 5-ring PCF shown in Figure 8. The silica index was modeled using the known Sellmeier equation whereas the index of the holes was equal to 1 (air) to 1.1. After the PCF, the excited modes reach another solid piece of glass, that is, the other collapsed end of PCF. They will thus further diffract and will be recombined into core mode through the filtering of the subsequent SMF or PCF. Since the modes propagate at different phase velocities, thus in a certain length of PCF the modes accumulate a differential phase shift. The phase velocities and the phase difference are wavelength-dependent; therefore, the optical power transmitted (or reflected) by the device will be maximum at certain wavelengths and minimum at others.

The different configurations reported so far are illustrated in Figure 8. One of the first architectures that was demonstrated consisted of a PCF with two collapsed regions separated a few centimeters $[50,51]$. Later on a short section of PCF longitudinally sandwiched between standard single mode fibers was proposed $[52,53]$. The advantage of this configuration is that the modal properties of the PCF are exploited but the interrogation is carried out with conventional optical fibers, thus leading to more costeffective interferometers. More recently, a stub of PCF with cleaved end fusion spliced at the distal end of a single mode fiber was demonstrated $[54,55]$. In this case the interferometer operates in reflection and a single circulator or coupler is needed to interrogate the device. It is important to point out that the properties, and consequently the applications of the interferometer depend on the type of PCF.

The interferometers fabricated via micro-holes collapsing exhibit truly sinusoidal and stable interference spectra which are observed over a broad wavelength range $(\sim 800 \mathrm{~nm})$. Figure 10, for example, shows the interference patterns observed in devices operating in transmission and reflection mode. The period or fringe spacing $(\Delta \lambda)$ of these interferometers is $\Delta \lambda \approx \lambda^{2} /\left(\Delta n_{\text {eff }} L\right)$, being $\Delta n_{\text {eff }}$ the effective refractive index difference between the modes participating in the interference. Such modes can be two core modes or a core and a cladding mode, depending on the PCF employed. $L$ is the length of PCF or the length between collapsed regions, see Figure 8 . The period or fringe spacing in these 


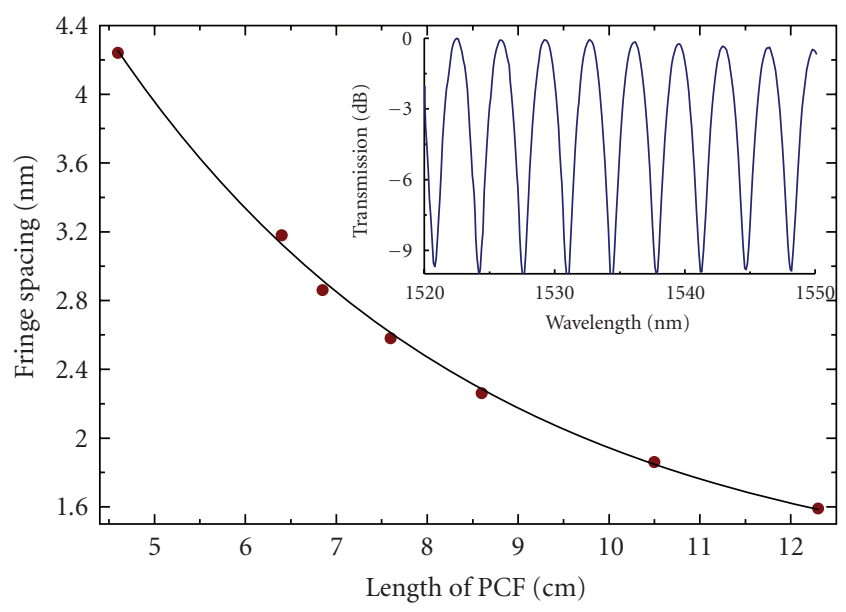

(a)

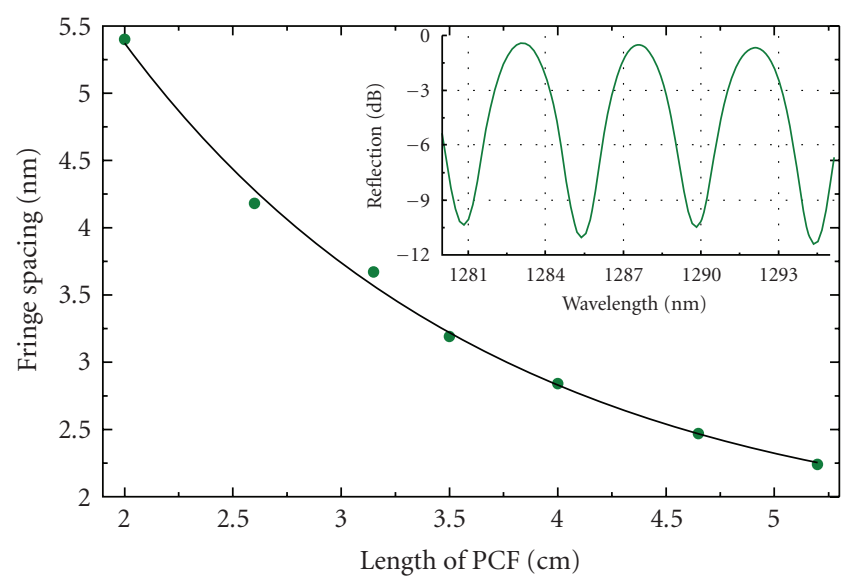

- Experimental data

- Fit

(b)

Figure 10: (a) Fringe spacing as a function of the length of PCF observed in interferometers operating in transmission mode. The inset shows the spectrum of an interferometer with $L=75 \mathrm{~mm}$. (b) Period as a function of $L$ observed in devices operating in reflection mode. The inset shows the spectrum of a device with $L=26 \mathrm{~mm}$. In both cases the fringe spacing was measured at $1550 \mathrm{~nm}$.

interferometers can be controlled easily with the length of PCF between the two splices.

PCF interferometers fabricated via microhole collapsed exhibit some important features that are important for optical sensing. In general the performance, stability, and temperature dependence of a modal interferometer depend critically on the element that excites and recombines the modes. In interferometers built via microhole collapsing the excitation and recombination of modes is carried out with permanent and stable splices. These do not degrade over time or with temperature, thus interferometers with high stability are achievable. Their thermal sensitivity depends on the type of PCF one uses as well as the length of fiber. It is low, it can go from $\sim 5$ to $\sim 10 \mathrm{pm} /{ }^{\circ} \mathrm{C}$. In some applications no temperature compensation may be needed. For example,

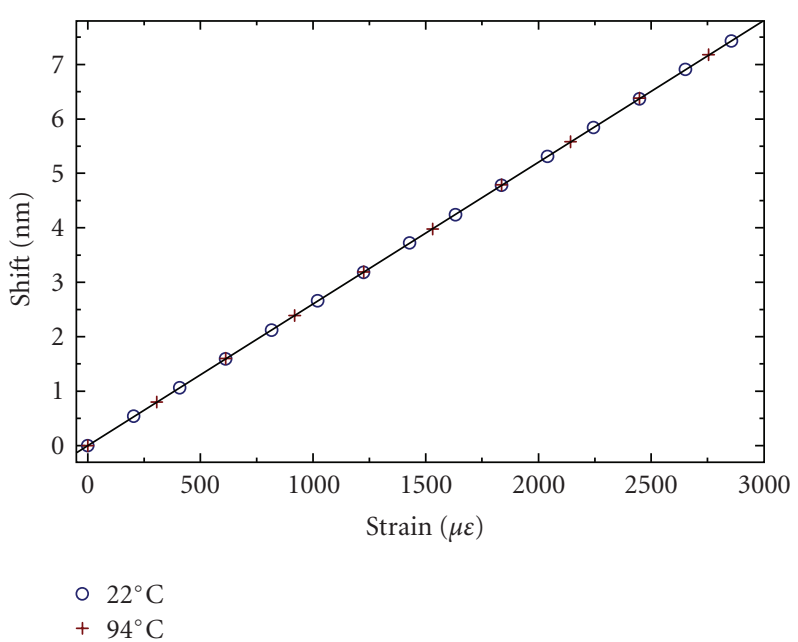

FIGURE 11: Shift of the interference pattern as a function of strain observed at room temperature and $94^{\circ} \mathrm{C}$. The measurements were carried out at $1550 \mathrm{~nm}$ with a device with $L=10.53 \mathrm{~mm}$.

in Figure 11 we show the shift as a function of the applied strain observed in a device fabricated with $10.53 \mathrm{~cm}$ of 5ring PCF at different temperatures. Note that the behavior of the interferometer is similar which demonstrates that no temperature compensation is required. The strain sensitivity of this device is $2.6 \mathrm{pm} / \mu \varepsilon$ which is higher than that of other PCF interferometric strain sensors.

As mentioned before, the features of the interferometers depend on the microstructure of the PCF. For example, by splicing a PCF consisting of seven rings of holes (LMA8 from Crystal Fibre), then core and cladding modes are excited $[53,54]$. Cladding modes, for example, are sensitive to the external environment, thus making possible the detection of samples present on the surface of the PCF. In this case the interaction is solely with the cladding modes since the core mode is isolated from the external environment. The interaction of the cladding modes with the external index changes $\Delta n_{\text {eff }}$, and consequently the phase difference. As a result, the position of the interference peaks and valleys change, or equivalently the interference pattern shifts. Therefore by monitoring the interference pattern wavelength shift one can infer the external refractive index and its changes. For example, the inset of Figure 12 shows the transmission spectra over $30 \mathrm{~nm}$ observed in an interferometer fabricated with $L=17 \mathrm{~mm}$ when it was in air (refractive index of 1) and in a liquid with index of 1.330. The figures also show the shifts measured as a function the external index. It is worth noting that the range of refractive indices that can be measured is quite broad, from 1.33 (aqueous environments) to 1.44 (biomolecules). The resolution of is estimated to be $\sim 2.9 \times 10^{-4}$ in the 1.40 1.44 range [53].

When the interferometers operate in reflection mode novel sensing applications are possible. For example, an in-reflection interferometer fabricated by fusion splicing a stub of 5-ring PCF (see Figure 8) at the distal end of a standard optical fiber (Corning SMF-28) can be used 


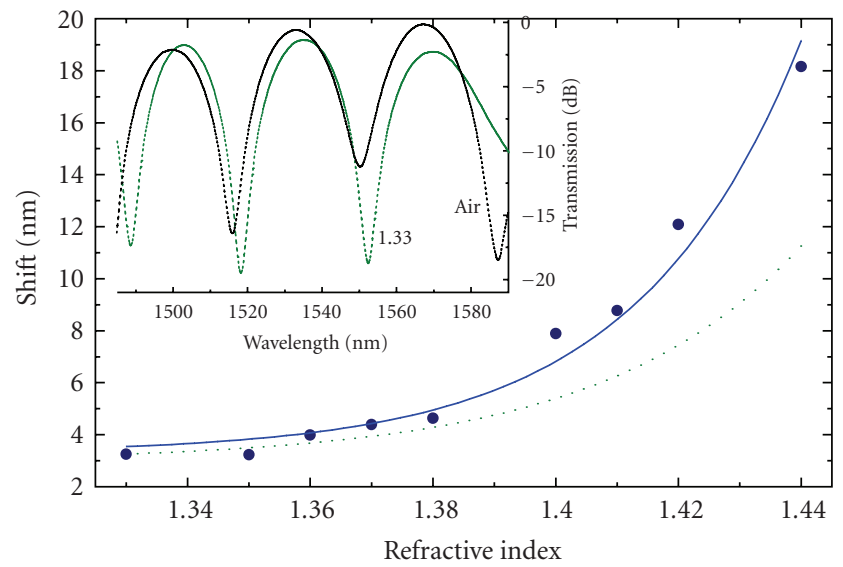

Figure 12: Shift of the interference pattern as a function of the external refractive index observed in a device with $L=17 \mathrm{~mm}$. The circles are experimental data and the continuous line is a fitting to the data. The measurements were carried out in the $1490-1590 \mathrm{~nm}$ wavelength range.

to detect different volatile organic compounds at room temperature. The key point here is to leave the voids of the PCF open to allow infiltration of some gases, chemical vapor, or molecules. The interaction of the compounds with the interfering modes takes place in the first rings of voids which have a total volume in the picolitters range [55]. Figure 13 for example shows the shift of an interferometer in which $L$ was $37 \mathrm{~mm}$ when exposed to vapor of acetone in closed and open chamber. Figure 13(a) was collected by evaporating $5 \mu \mathrm{l}$ of acetone in a closed container. The shift increases owing to the presence of the vapor. The fact that the curve plateaus indicates that the chamber was properly sealed. Figure 13(b) was collected by evaporating $75 \mu \mathrm{L}$ of acetone in an open container. Note that the interferometer saturates owing to the limited volume of the voids. They can house a maximum number of molecules. The interferometer slowly returns to its original position which indicates the reversibility of the devices. The response time of the device is long because the experiments were carried at normal conditions. In addition, the infiltration of the acetone vapor into the microscopic voids of the PCF is a diffusion process which is typically slow.

We would like to point out that the combination of different fibers to form interferometers has been investigated during many years [58-61]. In these cases the splicing is critical since it determines the splitting and/or recombination of the interfering beams or mores. The selection of the fiber in which two modes are excited is also crucial.

\section{Conclusions and Outlook}

Photonic crystal fibers are at the forefront of fiber optics technology. These fibers offer different possibilities for the fiber optic sensing field as demonstrated by different works reported in the past few years. PCFs offer different alternatives for the fabrication of modal interferometers,

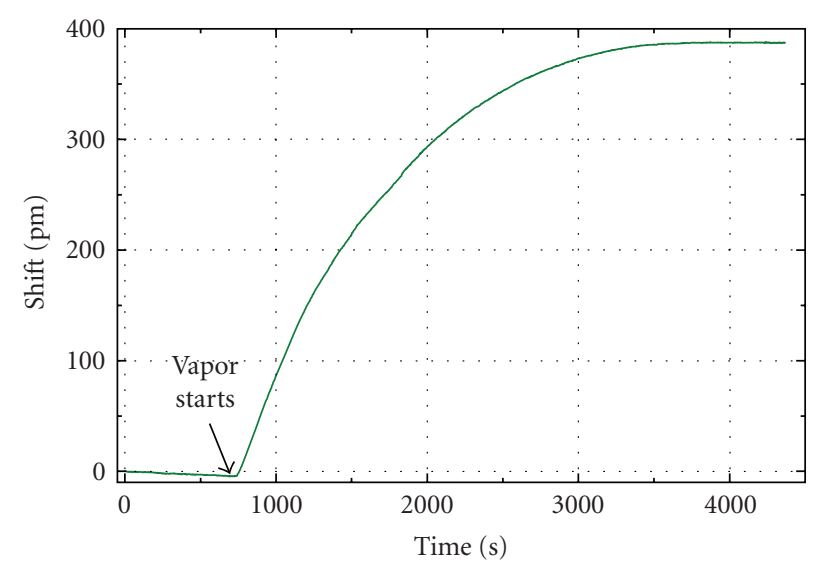

(a)

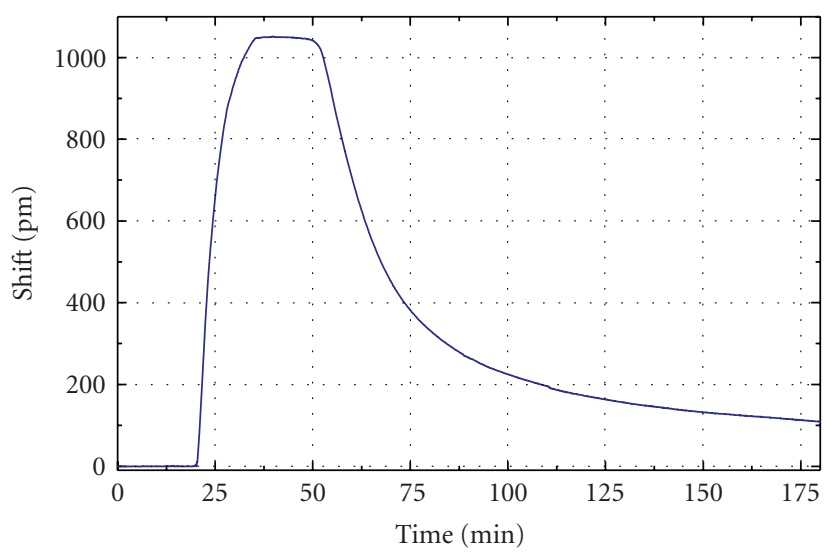

(b)

FIGURE 13: Shift observed in a $37 \mathrm{~mm}$-long interferometer when exposed to acetone vapor in closed (LHS) and open container (RHS). The measurements were carried out at $1310 \mathrm{~nm}$.

Sagnac-, and Mach-Zehnder- or Michelson-like ones. Most of them can also be implemented with standard optical fiber but PCFs give them important advantages such as low thermal sensitivity, broad operation wavelength range, or high stability over time. However, other cases have no counterparts in conventional fibers. Interferometers that exploit the diffraction caused by a short region with collapsed voids, for example, are completely new. In this case the holey microstructure of the PCF is fully exploited. It will, therefore be no surprise if some of the interferometers reviewed in this paper end as innovative commercial products in the near future or at least represent new solutions to old problems (temperature compensation) in optical sensing.

\section{Acknowledgments}

This work was carried out with the financial support of the Spanish MEC through grant TEC2006-10665/MIC and the "Ramón y Cajal" program. The authors are grateful to Vladimir P. Minkovich and D. Monzón-Hernández for their valuable help. 


\section{References}

[1] D. A. Jackson and J. D. C. Jones, "Interferometers," in Optical Fiber Sensors: Systems and Applications, B. Culshaw and J. Dakin, Eds., pp. 239-280, Artech House, Norwood, Mass, USA, 1989.

[2] J. D. C. Jones, "Interferometry and polarimetry for optical sensing," in Handbook of Optical Fibre Sensing Technology, J. M. López-Higuera, Ed., pp. 227-245, John Wiley \& Sons, West Sussex, UK, 2002.

[3] F. T. S. Yu and S. Yin, Eds., Fiber Optic Sensors, Marcel \& Dekker, New York, NY, USA, 2002.

[4] P. St. J. Russell, "Photonic-crystal fibers," IEEE Journal of Lightwave Technology, vol. 24, pp. 4729-4749, 2006.

[5] A. Bjarklev, J. Broeng, and A. S. Bjarklev, Photonic Crystal Fibres, Kluwer Academic Publishers, Boston, Mass, USA, 2003.

[6] O. Frazao, J. L. Santos, F. M. Araujo, and L. A. Ferreira, "Optical sensing with photonic crystal fibers," Laser \& Photonics Reviews, vol. 2, pp. 449-459, 2008.

[7] W. N. MacPherson, M. J. Gander, R. McBride, et al., "Remotely addressed optical fibre curvature sensor using multicore photonic crystal fibre," Optics Communications, vol. 193, no. 1-6, pp. 97-104, 2001.

[8] D. Káčik, I. Turek, I. Martinček, J. Canning, N. A. Issa, and K. Lyytikäinen, "Intermodal interference in a photonic crystal fibre," Optics Express, vol. 12, no. 15, pp. 3465-3470, 2004.

[9] J. Ju, W. Jin, and M. S. Demokan, "Two-mode operation in highly birefringent photonic crystal fiber," IEEE Photonics Technology Letters, vol. 16, no. 11, pp. 2472-2474, 2004.

[10] M. R. Layton and J. A. Bucaro, "Optical fiber acoustic sensor utilizing mode-mode interference," Applied Optics, vol. 18, no. 5, pp. 666-670, 1979.

[11] M. Spajer, B. Carquille, and H. Maillotte, "Application of intermodal interference to fibre sensors," Optics Communications, vol. 60 , no. 5 , pp. 261-264, 1986 .

[12] T. A. Eftimov and W. J. Bock, "Sensing with a $\mathrm{LP}_{01}-\mathrm{LP}_{02}$ intermodal interferometer," Journal of Lightwave Technology, vol. 11, no. 12, pp. 2150-2156, 1993.

[13] T.-J. Chen, "A novel two-mode fiber-optic interferometer based on $\mathrm{HE}_{11}-\mathrm{TE}_{01}$ modal interference utilizing a liquidcrystal-clad fiber modal filter," Optics Communications, vol. 261, no. 1, pp. 43-50, 2006.

[14] W. Jin, Z. Wang, and J. Ju, "Two-mode photonic crystal fibers," Optics Express, vol. 13, no. 6, pp. 2082-2088, 2005.

[15] C.-L. Zhao, X. Yang, C. Lu, W. Jin, and M. S. Demokan, "Temperature-insensitive interferometer using a highly birefringent photonic crystal fiber loop mirror," IEEE Photonics Technology Letters, vol. 16, no. 11, pp. 2535-2537, 2004.

[16] D.-H. Kim and J. U. Kang, "Sagnac loop interferometer based on polarization maintaining photonic crystal fiber with reduced temperature sensitivity," Optics Express, vol. 12, no. 19, pp. 4490-4495, 2004.

[17] X. Dong, H. Y. Tam, and P. Shum, “Temperature-insensitive strain sensor with polarization-maintaining photonics crystal fiber based on Sagnac interferometer," Applied Physics Letters, vol. 90, no. 15, Article ID 151113, 3 pages, 2007.

[18] O. Frazao, J. M. Baptista, and J. L. Santos, "Temperature independent strain sensor based on a Hi-Bi photonic crystal fiber loop mirror," IEEE Journal Sensors, vol. 7, pp. 1453-1455, 2007.

[19] H. Y. Fu, H. Y. Tam, L.-Y. Shao, et al., "Pressure sensor realized with polarization-maintaining photonic crystal fiberbased Sagnac interferometer," Applied Optics, vol. 47, no. 15, pp. 2835-2839, 2008.
[20] O. Frazão, J. Baptista, J. L. Santos, and P. Roy, "Curvature sensor using a highly birefringent photonic crystal fiber with two asymmetric hole regions in a Sagnac interferometer," Applied Optics, vol. 47, no. 13, pp. 2520-2523, 2008.

[21] G. Kim, T. Cho, K. Hwang, et al., "Strain and temperature sensitivities of an elliptical hollow-core photonic bandgap fiber based on Sagnac interferometer," Optics Express, vol. 17, no. 4, pp. 2481-2486, 2009.

[22] V. Bhatia and A. M. Vengsarkar, "Optical fiber long-period grating sensors," Optics Letters, vol. 21, no. 9, pp. 692-694, 1996.

[23] B. H. Lee and J. Nishii, "Self-interference of long-period fibre grating and its application as temperature sensor," Electronics Letters, vol. 34, no. 21, pp. 2059-2060, 1998.

[24] B. H. Lee and J. Nishii, "Dependence of fringe spacing on the grating separation in a long-period fiber grating pair," Applied Optics, vol. 38, no. 16, pp. 3450-3459, 1999.

[25] O. Duhem, J. F. Henninot, and M. Douay, "Study of in fiber Mach-Zehnder interferometer based on two spaced 3-dB long period gratings surrounded by a refractive index higher than that of silica," Optics Communications, vol. 180, no. 4, pp. 255262, 2000.

[26] T. Allsop, R. Reeves, D. J. Webb, I. Bennion, and R. Neal, "A high sensitivity refractometer based upon a long period grating Mach-Zehnder interferometer," Review of Scientific Instruments, vol. 73, no. 4, pp. 1702-1705, 2002.

[27] S. W. James, I. Ishaq, G. J. Ashwell, and R. P. Tatam, "Cascaded long-period gratings with nanostructured coatings," Optics Letters, vol. 30, no. 17, pp. 2197-2199, 2005.

[28] I. D. Villar, M. Achaerandio, F. J. Arregui, and I. R. Matias, "Generation of selective fringes with cascaded long-period gratings," IEEE Photonics Technology Letters, vol. 18, no. 13, pp. 1412-1414, 2006.

[29] P. L. Swart, "Long-period grating Michelson refractometric sensor," Measurement Science and Technology, vol. 15, no. 8, pp. 1576-1580, 2004.

[30] D. W. Kim, Y. Zhang, K. L. Cooper, and A. Wang, "In-fiber reflection mode interferometer based on a long-period grating for external refractive-index measurement," Applied Optics, vol. 44, no. 26, pp. 5368-5373, 2005.

[31] J. H. Lim, H. S. Jang, K. S. Lee, J. C. Kim, and B. H. Lee, "Mach-Zehnder interferometer formed in a photonic crystal fiber based on a pair of long-period fiber gratings," Optics Letters, vol. 29, no. 4, pp. 346-348, 2004.

[32] X. Yu, P. Shum, and X. Dong, "Photonic-crystal-fiber-based Mach-Zehnder interferometer using long-period gratings," Microwave and Optical Technology Letters, vol. 48, no. 7, pp. 1379-1383, 2006.

[33] H. Y. Choi, K. S. Park, and B. H. Lee, "Photonic crystal fiber interferometer composed of a long period fiber grating and one point collapsing of air holes," Optics Letters, vol. 33, no. 8, pp. 812-814, 2008.

[34] J. Ju, W. Jin, and H. L. Ho, "Compact in-fiber interferometer formed by long-period gratings in photonic crystal fiber," IEEE Photonics Technology Letters, vol. 20, no. 23, pp. 18991901, 2008.

[35] S. T. Huntington, J. Katsifolis, B. C. Gibson, et al., "Retaining and characterizing nano-structure within tapered air-silica structured optical fibers," Optics Express, vol. 11, pp. 98-104, 2003.

[36] H. C. Nguyen, B. T. Kuhlmey, E. C. Magi, et al., "Tapered photonic crystal fibers: properties, characterization and applications," Applied Physics B, vol. 81, pp. 377-387, 2005. 
[37] J. Hu, B. S. Marks, C. R. Menyuk, et al., "Pulse compression using a tapered microstructure optical fiber," Optics Express, vol. 14, no. 9, pp. 4026-4036, 2006.

[38] S. Laflamme, S. Lacroix, J. Bures, and X. Daxhelet, "Understanding power leakage in tapered solid core microstructured fibers," Optics Express, vol. 15, no. 2, pp. 387-396, 2007.

[39] M.-L. V. Tse, P. Horak, F. Poletti, and D. J. Richardson, "Designing tapered holey fibers for soliton compression," IEEE Journal of Quantum Electronics, vol. 44, no. 2, pp. 192-198, 2008.

[40] V. P. Minkovich, J. Villatoro, D. Monzón-Hernández, S. Calixto, A. B. Sotsky, and L. I. Sotskaya, "Holey fiber tapers with resonance transmission for high-resolution refractive index sensing," Optics Express, vol. 13, no. 19, pp. 7609-7614, 2005.

[41] J. Villatoro, V. P. Minkovich, and D. Monzón-Hernández, "Temperature-independent strain sensor made from tapered holey optical fiber," Optics Letters, vol. 31, no. 3, pp. 305-307, 2006.

[42] D. Monzón-Hernández, V. P. Minkovich, and J. Villatoro, "High-temperature sensing with tapers made of microstructured optical fiber," IEEE Photonics Technology Letters, vol. 18, pp. 511-513, 2006.

[43] V. P. Minkovich, D. Monzón-Hernández, J. Villatoro, and G. Badenes, "Microstructured optical fiber coated with thin films for gas and chemical sensing," Optics Express, vol. 14, no. 18, pp. 8413-8418, 2006.

[44] V. P. Minkovich, D. Monzón-Hernández, J. Villatoro, A. B. Sotsky, and L. I. Sotskaya, "Modeling of holey fiber tapers with selective transmission for sensor applications," Journal of Lightwave Technology, vol. 24, no. 11, pp. 4319-4328, 2006.

[45] D. Monzón-Hernández, V. P. Minkovich, J. Villatoro, M. P. Kreuzer, and G. Badenes, "Photonic crystal fiber microtaper supporting two selective higher-order modes with high sensitivity to gas molecules," Applied Physics Letters, vol. 93, no. 8, Article ID 081106, 3 pages, 2008.

[46] X. Daxhelet, J. Bures, and R. Maciejko, "Temperatureindependent all-fiber modal interferometer," Optical Fiber Technology, vol. 1, no. 4, pp. 373-376, 1995.

[47] K. Q. Kieu and M. Mansuripur, "Biconical fiber taper sensors," IEEE Photonics Technology Letters, vol. 18, pp. 2239-2241, 2006.

[48] L. Yuan, J. Yang, Z. Liu, and J. Sun, "In-fiber integrated Michelson interferometer," Optics Letters, vol. 31, no. 18, pp. 2692-2694, 2006.

[49] A. Ozcan, A. Tewary, M. J. F. Digonnet, and G. S. Kino, "Observation of mode coupling in bitapered air-core photonic bandgap fibers," Optics Communications, vol. 271, no. 2, pp. 391-395, 2007.

[50] J. Villatoro, V. P. Minkovich, V. Pruneri, and G. Badenes, "Simple all-microstructured-optical-fiber interferometer built via fusion splicing," Optics Express, vol. 15, no. 4, pp. 14911496, 2007.

[51] H. Y. Choi, M. J. Kim, and B. H. Lee, "All-fiber Mach-Zehnder type interferometers formed in photonic crystal fiber," Optics Express, vol. 15, no. 9, pp. 5711-5720, 2007.

[52] J. Villatoro, V. Finazzi, V. P. Minkovich, V. Pruneri, and G. Badenes, "Temperature-insensitive photonic crystal fiber interferometer for absolute strain sensing," Applied Physics Letters, vol. 91, no. 9, Article ID 091109, 3 pages, 2007.

[53] R. Jha, J. Villatoro, G. Badenes, and V. Pruneri, "Refractometry based on a photonic crystal fiber interferometer," Optics Letters, vol. 34, no. 5, pp. 617-619, 2009.
[54] R. Jha, J. Villatoro, and G. Badenes, "Ultrastable in reflection photonic crystal fiber modal interferometer for accurate refractive index sensing," Applied Physics Letters, vol. 93, no. 19, Article ID 191106, 3 pages, 2008.

[55] J. Villatoro, M. P. Kreuzer, R. Jha, et al., "Photonic crystal fiber interferometer for chemical vapor detection with high sensitivity," Optics Express, vol. 17, no. 3, pp. 1447-1453, 2009.

[56] B. Bourliaguet, C. Paré, F. Émond, A. Croteau, A. Proulx, and R. Vallée, "Microstructured fiber splicing," Optics Express, vol. 11, no. 25, pp. 3412-3417, 2003.

[57] A. D. Yablon and R. T. Bise, "Low-loss high-strength microstructured fiber fusion splices using GRIN fiber lenses," IEEE Photonics Technology Letters, vol. 17, no. 1, pp. 118-120, 2005.

[58] J. Canning and A. L. G. Carter, "Modal interferometer for in situ measurements of induced core index change in optical fibers," Optics Letters, vol. 22, no. 8, pp. 561-563, 1997.

[59] Q. Li, C.-H. Lin, P.-Y. Tseng, and H. P. Lee, "Demonstration of high extinction ratio modal interference in a two-mode fiber and its applications for all-fiber comb filter and hightemperature sensor," Optics Communications, vol. 250, no. 46, pp. 280-285, 2005.

[60] Q. Wang and G. Farrell, "All-fiber multimode-interferencebased refractometer sensor: proposal and design," Optics Letters, vol. 31, no. 3, pp. 317-319, 2006.

[61] Y. Jung, S. Kim, D. Lee, and K. Oh, "Compact three segmented multimode fibre modal interferometer for high sensitivity refractive-index measurement," Measurement Science and Technology, vol. 17, no. 5, pp. 1129-1133, 2006. 

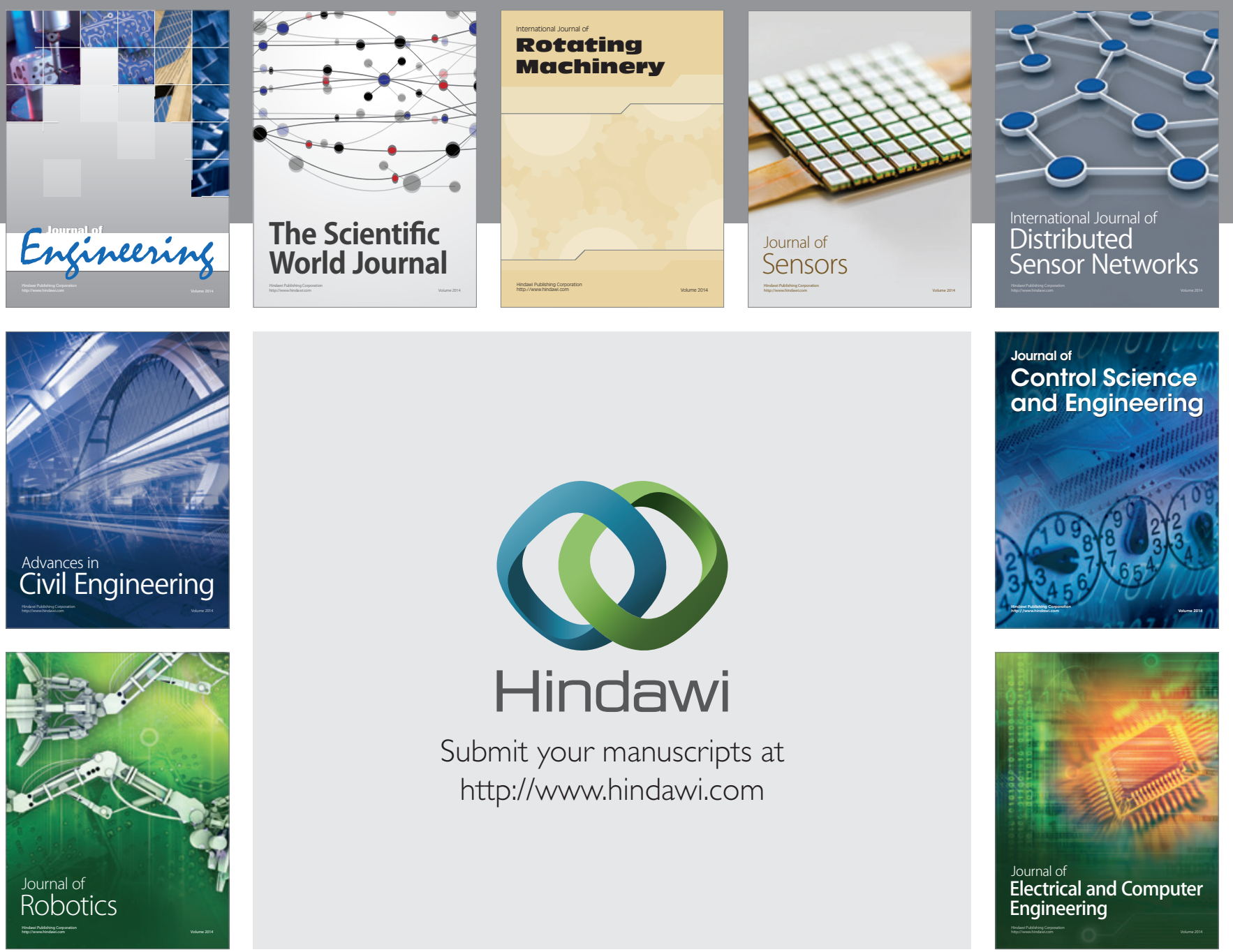

Submit your manuscripts at

http://www.hindawi.com
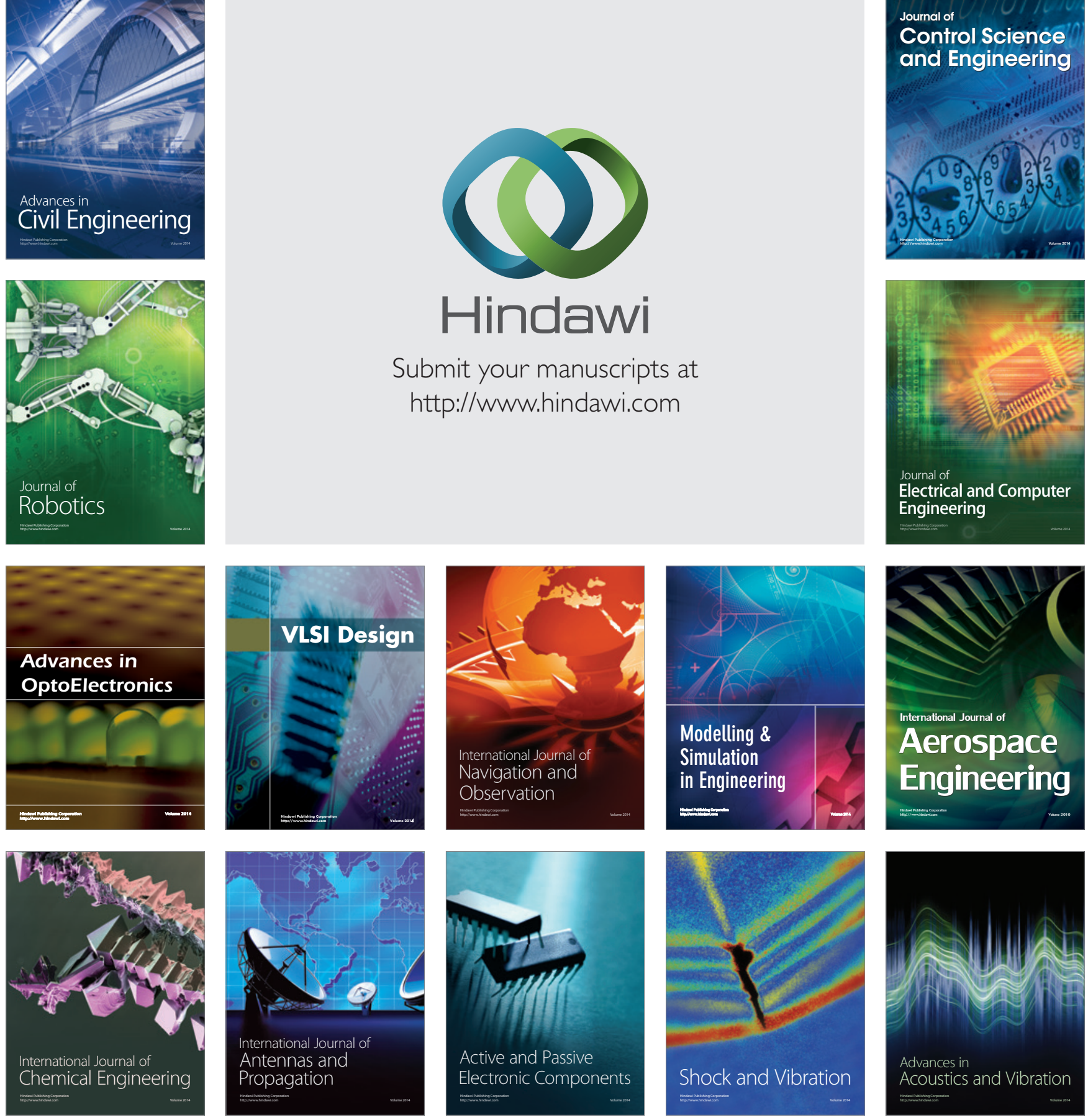